\title{
The Effect of Educational Intervention in Promoting Safe Behaviors in a Sample of Iranian Primary School Students: An Application of the Health Belief Model
}

\author{
Maryam Ghavami ${ }^{1}$, Tayebeh Fasihi Harandy ${ }^{2} \&$ Kourosh Kabir ${ }^{3}$ \\ ${ }^{1}$ Health Education Department in Alborz University of Medical Sciences, Karaj, Iran \\ ${ }^{2}$ Social Determinants of Health Research Center, Alborz University of Medical Sciences, Karaj, Iran \\ ${ }^{3}$ Department of Community Medicine, School of Medicine, Alborz University of Medical Sciences, Karaj, Iran \\ Correspondence: Tayebeh Fasihi Harandy, Assistant Prof., Social Determinants of Health Research Center, \\ Alborz University of Medical Sciences, Karaj, Iran. E-mail: tfasihih@gmail.com
}

Received: January 23, 2016 Accepted: March 9, 2016 Online Published: March 31, 2016

doi:10.5539/gjhs.v8n11p242 URL: http://dx.doi.org/10.5539/gjhs.v8n11p242

\begin{abstract}
Purpose: Road traffic accidents account for the biggest mortality rate in Iran and children are among the main victims of these accidents. The present study was conducted to assess the effect of an educational intervention based on the Health Belief Model on behaviors associated with obeying traffic regulations in primary school students during the academic year 2014-15 in Khorramabad, Iran.

Methods: The present quasi-experimental has a pre-post-intervention design and was conducted on 106 sixthgrade primary school students selected through multi-stage sampling and divided into an intervention and a control group. The students were assessed before, a week after and two months after the intervention. Data were collected using a two-part questionnaire containing a demographic section and a section with items on the constructs of the Health Belief Model. The data obtained were then analyzed in SPSS- 20 .
\end{abstract}

Results: The mean scores of perceived susceptibility, perceived severity, perceived benefits, perceived barriers, self-efficacy and cues to action significantly improved in the intervention group compared to the control group $(\mathrm{P}<0.01)$.

Conclusion: An educational intervention based the Health Belief Model managed to effectively improve behaviors associated with obeying traffic regulations.

Keywords: educational intervention, traffic, safe behaviors, primary school students

\section{Introduction}

Accidents have become major global economic, social and health risk factors across the world today (Sharma, 2008). Accident is taken to refer to an unpredictable event that often occurs during transport and in the workplace, the living habitat or recreational centers and causes harm (Brussoni et al., 2015). Road traffic accidents claim an annual of 1.3 million lives and injure 20 to 50 million across the world (Puvanachandra et al., 2012), account for the biggest mortality rate due to unintentional injuries ( $\mathrm{Sim} \& \mathrm{Ng}, 2005$ ) and are expected to rank as the fifth leading cause of death by 2030 (Fathi Shamsi, Shamsi, Khorsandi, \& Ranjbaran, 2015). In Iran, road traffic accidents are the cause of an annual of more than 48,000 deaths, 300 thousand hospital stays exceeding one day and nearly 3 million injuries requiring outpatient care (Farhadi, Roshanaei, Bashirian, \& Rezapoor Shahkelaei, 2014).

The 14-million student population of the country and the demands dictated by their young age and inexperience make students prone to accidents and injuries at school and during the school commute (Vojdani Aram, Roshanaei, Hazavei, Karimi Shahanjarini, \& Rezapoor Shahkelaei, 2014). As one of the most vulnerable groups of the population, children are at a greater risk of road accidents due to their inexperience, frisky and impetuous nature and the lack of distance estimation abilities for oncoming vehicles and unfamiliarity with the risks these accidents pose (Vojdani-Aram, Roshanaei, Hazavehei, Karimi-Shahanjarini, \& Rezapur-Shahkolai, 2015). Several studies have shown that children under the age of 11 are more prone to sidewalk injuries while older children are more prone to bicycle and motorcycle injuries (Jaber-Gaderi et al., 2008; Nazari et al., 2008; Zargar, 
Sayyer Roudsarib, Shadman, \& Kavianie and Payam Tarighib, 2003).

Accepting accidents as preventable events has caused the adoption of preventive measures and therefore the reduction of accident-related mortality rates in some countries (Naghavi \& Akbari, 2002). Although many factors are involved in causing accidents, people and their unsafe conduct (not fastening the seat belt, not wearing helmets, etc.) account for the majority of accidents (Rezapur Shahkolai, Farhadi, Bashirian, \& Roshanaei, 2013). Several studies have shown that education is one of the main issues required for resolving traffic problems (Jaber-Gaderi et al., 2008; Jackson, 1997; Nazari et al., 2008; Nordberg, 2000) and that all age groups should be trained in order to improve the culture of safety in the community. This improvement requires the implementation of appropriate educational measures, the promotion of safe conduct and the building of a standard road safety culture (Rezapur Shahkolai et al., 2013). The Health Belief Model (HBM) provides an extensive framework for planning and implementing educational interventions (Hazavehei, Taghdisi, \& Saidi, 2007) and involves extensive empirical support for predicting a wide range of health and safety behaviors, especially in the case of intentional and unintentional accidents (Cao, Chen, \& Wang, 2014; Cheraghi, Poorolajal, Hazavehi, \& Rezapur-Shahkolai, 2014; Hazavehei et al., 2007; Rezapur Shahkolai et al., 2013; Vojdani-Aram et al., 2015). This model focuses on the individual's behavior as a function of his knowledge and attitude toward behavior, according to the theoretical bases which the individual adopts preventive health behaviors only when he is convinced of factors such as perceived susceptibility (the individual's perception of his vulnerability or predisposition to a disease or health condition), perceived severity (his belief in the severity of a condition and its consequences), perceived benefits (his belief in the effectiveness of adopting preventive behaviors), perceived barriers (his belief in the negative aspects of the behaviors), cues to action (environmental stimuli such as health messages, people's suggestions, etc.) and self-efficacy (the individual's trust in his own ability for the successful performance of the intended behavior) (Rosenstock \& Stretcher, 2008). Using this model enables health educators to help individuals develop a realistic perspective to danger (Cheraghi et al., 2014). The present study was conducted to promote safe behaviors in students.

\section{Materials and Methods}

The present randomized clinical trial was conducted on 106 sixth-grade primary school students in Khorramabad to determine the effect of an educational intervention based on the HBM. The study inclusion criteria consisted of having no known mental disorders and being willing to participate in the study.

Sampling was performed through the stratified multistage method. In the first stage, the schools were categorized based on their education districts (districts 1 and 2 of the city) and their students' gender (boys' school and girls' school). In each category, four schools were randomly selected for undergoing the intervention (two boys' schools and two girls' schools) and four as the controls (two boys' schools and two girls' schools). For the last stage, samples were selected randomly from the sixth-grade students in each school.

To assess the performance of safe behaviors and the constructs of the HBM, the traffic regulation behaviors questionnaire was used, which has a confirmed validity and reliability according to previous studies (Heshmati et al., 2013). This questionnaire contains a demographic details' section with 7 items, an HBM constructs' section with a total of 26 items divided between perceived susceptibility (4 items), perceived severity (6 items), perceived benefits ( 5 items), perceived barriers (6 items) and self-efficacy (5 items), which are scored based on a 5-point Likert scale with options ranging from totally agree to totally disagree and which are given scores of 0 to 4 , with each construct worth 100 points, and a final section on behavior, which has 12 items, each receiving a score from 0 to 4 for the answers of "always, often, occasionally, rarely and never". The reliability of this tool was assessed once again through measuring its internal consistency, which gave a Cronbach's alpha of 0.77.

After obtaining permission from the authorities at the Ministry of Education for carrying out the proposed intervention and after the students' parents gave their informed consents for the participation of their children in the study, the self-reporting pretest was completed. After analyzing the initial data, the educational content of the intervention was developed in three domains, including cognitive, emotional and behavioral domains, and based on the general objectives of the research and the HBM, and was focused on introducing the students to traffic regulations. The educational intervention was then performed over three sessions of 30 to 45 minutes using group discussion and Q\&A. Visual cards were used as cues to action. To observe the ethical codes of conducting clinical research, the control group also received educational pamphlets on traffic regulations at the end of the intervention.

Data were analyzed in SPSS using the independent t-test, the one-way analysis of variance, the repeated measures ANOVA and the regression analysis. 


\section{Results}

The 106 students examined were at an age range of 11 to 13 and had a mean age of $12.19 \pm 0.59$ years in the intervention group and $12.26 \pm 0.66$ years in the control group.

Before the intervention, the two groups matched in terms of their demographic variables, including their parents' age, level of education and occupation and their household size.

The one-way analysis of variance on the intra- and extra-group factors showed significant differences between the students in their perceived susceptibility $(0.002)$, perceived severity $(0.0001)$, perceived barriers $(0.0001)$, perceived benefits $(0.0001)$ and self-efficacy $(0.0001)$ in the pretest, posttest and the follow-up.

The results of the present study showed that behaviors associated with obeying traffic regulations were positively and significantly related with some of the constructs of the HBM, including perceived benefits, perceived barriers, perceived susceptibility and self-efficacy, while they were not related with the construct of perceived severity (Table 1).

Table 1. The correlation between the constructs of the HBM and behaviors associated with obeying traffic regulations

\begin{tabular}{llllll}
\hline Variances & $\begin{array}{l}\text { perceived } \\
\text { susceptibility }\end{array}$ & $\begin{array}{l}\text { perceived } \\
\text { severity }\end{array}$ & perceived benefits & perceived barriers & self-efficacy \\
\hline $\begin{array}{l}\text { safe } \\
\text { behaviors }\end{array}$ & $.29 * *$ & .14 & $.28^{* *}$ & $.30^{* *}$ & $.51^{* *}$ \\
\hline
\end{tabular}

$* * \mathrm{p}<0.1$.

The stepwise linear multivariate regression analysis showed a significant correlation between behaviors associated with obeying traffic regulations and the constructs of HBM (F was observed). In the first step of the regression analysis and the first model, of the $34.4 \%$ explanation coefficient of the criterion variable, self-efficacy was inserted into the equation as a predicting variable and had a coefficient of 0.505 and thus explained $25.5 \%$ of the variance in the criterion variable. In the second step and the second model, the addition of perceived susceptibility with $\beta=0.298$ explained $8.9 \%$ of the variance in behaviors associated with obeying traffic regulations. The other predicting variables were eliminated from the model due to their insignificant regression coefficients.

The adjusted squared regression correlation coefficient was $\mathrm{R}^{2}=.255$ in the first model and $\mathrm{R}^{2}=.089$ in the second model, and each standard deviation unit of change in perceived susceptibility meant a $50 \%$ change in the standard deviation of safe behaviors (Table 2). The correlation between the criterion variable and the predicting variables was $\mathrm{R}=.505$ in the first step and $\mathrm{R}=.587$ in the second step (Table 2).

Table 2. The regression analysis of the subscales of the HBM constructs and their relationship with behaviors associated with obeying traffic regulations

\begin{tabular}{|c|c|c|c|c|c|c|c|c|c|c|c|c|}
\hline variance & Model & $\mathrm{R}$ & $\mathrm{R} 2$ & $\begin{array}{l}\text { R2 } \\
\text { Change }\end{array}$ & $\mathrm{F}$ & $\mathrm{P}$ & Model & $\beta$ & $\begin{array}{l}\text { Std. } \\
\text { Error }\end{array}$ & Beta & $\mathrm{t}$ & $\mathrm{P}$ \\
\hline \multirow{5}{*}{$\begin{array}{l}\text { safe } \\
\text { behaviors }\end{array}$} & \multirow{2}{*}{1} & \multirow{2}{*}{.505} & \multirow{2}{*}{.255} & \multirow{2}{*}{.255} & \multirow{2}{*}{35.66} & \multirow{2}{*}{.001} & Constant & 18.7 & 2.61 & \multirow{2}{*}{.505} & 7.18 & .001 \\
\hline & & & & & & & self-efficacy & 1.14 & .19 & & 5.97 & .001 \\
\hline & \multirow{3}{*}{2} & \multirow{3}{*}{.587} & \multirow{3}{*}{.344} & \multirow{3}{*}{.089} & \multirow{3}{*}{27.01} & \multirow{3}{*}{.001} & Constant & 9.39 & 3.52 & \multirow{2}{*}{.511} & 2.67 & .009 \\
\hline & & & & & & & self-efficacy & 1.16 & .18 & & 6.41 & .001 \\
\hline & & & & & & & $\begin{array}{l}\text { perceived } \\
\text { susceptibility }\end{array}$ & .74 & .20 & .298 & 3.73 & .001 \\
\hline
\end{tabular}

Before carrying out the multivariate linear regression analysis, its assumptions were confirmed, including the normal distribution of the variables, the linear relationship between the criterion and the predicting variables and the homogeneity of the variance. The inter- and intra-participant analysis of variance with three repeated 
measurements of behaviors associated with obeying traffic regulations in the intervention group and the control group showed significant differences between the pretest, posttest and follow-up scores in the constructs of HBM (Table 3).

Table 3. The mean and standard deviation of the pretest, posttest and follow-up scores in the intervention and control groups

\begin{tabular}{|c|c|c|c|c|c|c|c|c|}
\hline \multirow[t]{2}{*}{ variances } & \multirow[t]{2}{*}{ groups } & \multicolumn{2}{|c|}{$\begin{array}{ll}\text { before } & \text { the } \\
\text { intervention } & \end{array}$} & \multicolumn{2}{|c|}{$\begin{array}{l}\text { a week after the } \\
\text { intervention }\end{array}$} & \multicolumn{2}{|c|}{$\begin{array}{l}\text { Two months after the } \\
\text { intervention }\end{array}$} & \multirow{2}{*}{$\begin{array}{l}\mathbf{P} \\
\text { Value }\end{array}$} \\
\hline & & $\mathbf{M}$ & SD & $\mathbf{M}$ & SD & M & SD & \\
\hline \multirow{2}{*}{$\begin{array}{l}\text { perceived } \\
\text { susceptibility }\end{array}$} & intervention & 12.96 & 3.07 & 15.04 & 1.87 & 15.21 & 1.47 & \multirow[t]{2}{*}{.001} \\
\hline & control & 12.04 & 2.84 & 12.68 & 2.71 & 12.19 & 3.30 & \\
\hline \multirow{2}{*}{ perceived severity } & intervention & 14.21 & 4.69 & 21.62 & 3.83 & 22.32 & 2.30 & \multirow[t]{2}{*}{.001} \\
\hline & control & 15.04 & 4.25 & 16.62 & 4.25 & 16.21 & 3.39 & \\
\hline \multirow{2}{*}{ perceived benefits } & intervention & 15.32 & 3.19 & 17.83 & 2.11 & 18.62 & 1.94 & \multirow[t]{2}{*}{.001} \\
\hline & control & 14.47 & 3.21 & 14.94 & 3.12 & 14.98 & 3.12 & \\
\hline \multirow{2}{*}{$\begin{array}{l}\text { perceived } \\
\text { barriers }\end{array}$} & intervention & 16.45 & 4.20 & 20.19 & 3.04 & 20.62 & 2.64 & \multirow[t]{2}{*}{.001} \\
\hline & control & 15.42 & 4.30 & 15.62 & 3.78 & 14.91 & 3.35 & \\
\hline \multirow{2}{*}{ self-efficacy } & intervention & 13.13 & 3.42 & 18.23 & 2.38 & 18.30 & 2.41 & \multirow[t]{2}{*}{.001} \\
\hline & control & 13.38 & 3.10 & 13.58 & 3.08 & 13.28 & 3.77 & \\
\hline \multirow{2}{*}{ behavior } & intervention & 33.44 & 7.97 & 43.98 & 3.43 & 42.81 & 5.43 & \multirow[t]{2}{*}{.001} \\
\hline & control & 34.43 & 6.77 & 34.85 & 7.08 & 34.19 & 7.55 & \\
\hline
\end{tabular}

The results obtained showed a significant relationship between the intra-participant and inter-group scores of the factors in the pretest, posttest and follow-up stages, indicating the effectiveness of the educational intervention based on the HBM on all the behaviors associated with obeying traffic regulations in the students. A statistical power of more than 0.8 suggests the adequacy of the sample size.

\section{Discussion and Conclusion}

The results of the present study confirmed the effectiveness of an educational intervention based on the HBM in improving behaviors associated with obeying traffic regulations. These behaviors were found to be positively and significantly correlated with constructs including perceived benefits, perceived barriers, perceived susceptibility and self-efficacy, which is consistent with the results obtained in similar studies (Heshmati, Behnampour, Binaei, \& Khajavai, 2014; Morowatisharifabad, Momeni Sarvestani, Barkhordari Firoozabadi, \& Fallahzadeh, 2012; Sadeghnejad, 2014).

In line with the findings of other studies, constructs including perceived susceptibility and self-efficacy were good predictors for behaviors associated with obeying traffic regulations (Chen, Chiu, Chih, \& Yeh, 2015; Workagegn, Kiros, \& Abebe, 2015). The mean scores of perceived susceptibility and perceived severity increased after the intervention, which is consistent with the findings of several other studies (Cao et al., 2014; Farma, Jalili, Zareban, \& Pour, 2014; Joshi, Beckett, \& Macfarlane, 1994; Vojdani-Aram et al., 2015).The students' understanding that they could be at risk for traffic accidents raised their perceived susceptibility and led to their better adherence to behaviors associated with obeying traffic regulations. The educator's greater emphasis on the severity of the complications arising from having traffic injuries can significantly increase students' perceived severity. These results were in line with the results of other studies (Abood, Black, \& Feral, 2003; Jaber-Gaderi et al., 2008; Shojaezadeh, Naeimi, Noori, \& Khalili Haghverdi, 2015).

The mean score of perceived benefits showed a significant increase in the intervention group after the intervention, which, according to the researcher, was due to the appropriate educational methods used to explain the benefits of obeying traffic regulations, including group discussion and lecture.

The mean score of perceived barriers also increased significantly in the intervention group after the intervention, and the students developed a greater understanding of how to overcome the barriers. These results were in line 
with the results obtained in similar studies (Moodi, Mood, Sharifirad, Shahnazi, \& Sharifzadeh, 2011; Shojaezadeh et al., 2015).

The results of the present study suggest that an education focused on fostering the understanding and perception of the benefits of obeying traffic regulations as opposed to perceiving their barriers encourages students to make greater efforts for overcoming their perceived barriers to obeying these regulations.

The mean score of self-efficacy showed a significant increase in the intervention group after the intervention, which is in line with the results of similar studies (Jackson, 1997; Kealey et al., 2009; Shahnazi, Sabooteh, Sharifirad, Mirkarimi, \& Hassanzadeh, 2015; Tavassoli et al., 2015). Fostering the belief in the students that they can perform safe behaviors improves their chances of obeying traffic regulations (Farma et al., 2014; Hajian, Vakilian, Najabadi, Hosseini, \& Mirzaei, 2011; Jackson, 1997; Nazari et al., 2008; Rosenstock \& Stretcher, 2008).

The mean scores of safe behaviors associated with obeying traffic regulations increased significantly in the intervention group after the intervention, which is in line with the results of other studies.

The results of the present study are indicative of the positive effect of an educational intervention in cognitive, attitude and behavioral domains of obeying traffic regulations. Given that schools provide an appropriate setting for improving the understanding of the factors affecting students' behaviors, and since students act as a communication link between schools and parents, other educational interventions using different behavioral models are recommended to be conducted to help identify the factors affecting obeying traffic regulations and the building of a traffic culture in the society.

\section{Acknowledgements}

The present article is the result of an M.Sc. thesis in Health Education (under the code of ethics ABZUMS.REC.s.1394.16). The authors would like to express their gratitude to the University Research Deputy, the authorities at the Ministry of Education and all the headmasters, teachers and students of Khorramabad schools for their help in conducting this study.

\section{Conflict of Interest}

The authors declare that there is no conflict of interests regarding the publication of this paper.

\section{References}

Abood, D. A., Black, D. R., \& Feral, D. (2003). Nutrition education worksite intervention for university staff: Application of the health belief model. J Nut Educ Behav, 35(5), 260-267.

Brussoni, M., Gibbons, R., Gray, C., Ishikawa, T., Sandseter, E. B., Bienenstock, A., . . Tremblay, M. S. (2015). What is the Relationship between Risky Outdoor Play and Health in Children? A Systematic Review. Int $J$ Environ Res Public Health, 12(6), 6423-6454. http://dx.doi.org/10.3390/ijerph120606423

Cao, Z. J., Chen, Y., \& Wang, S. M. (2014). Health belief model based evaluation of school health education programme for injury prevention among high school students in the community context. BMC Pub Health, 14(1), 1-8. http://dx.doi.org/10.1186/1471-2458-14-26

Chen, C. H., Chiu, P. J., Chih, Y. C., \& Yeh, G. L. (2015). Determinants of influenza vaccination among young Taiwanese children. Vaccine, 33(16), 1993-1998. http://dx.doi.org/10.1016/j.vaccine.2015.01.032

Cheraghi, P., Poorolajal, J., Hazavehi, S. M., \& Rezapur-Shahkolai, F. (2014). Effect of educating mothers on injury prevention among children aged $<5$ years using the Health Belief Model: a randomized controlled trial. Public Health, 128(9), 825-830. http://dx.doi.org/10.1016/j.puhe.2014.06.017

Farhadi, Z., Roshanaei, G., Bashirian, M., \& Rezapoor Shahkelaei, F. (2014). [The effect of education programs for the prevention of damage caused by the events of the first secondary school students in the city Famenin Belief Model]. J Commun Health, 1(3), 9-11.

Farma, K. K., Jalili, Z., Zareban, I., \& Pour, M. S. (2014). Effect of education on preventive behaviors of breast cancer in female teachers of guidance schools of Zahedan city based on health belief model. $J$ Educ Health Promot, 3, 77. http://dx.doi.org/10.4103/2277-9531.139240

Fathi Shamsi, M., Shamsi, M., Khorsandi, M., \& Ranjbaran, M. (2015). [Structural measures for the prevention of accidents and incidents HBM children in the city of Khorramabad in 1393]. J Sci Arak Med Univ, 18(1), 69-77.

Hajian, S., Vakilian, K., Najabadi, K. M., Hosseini, J., \& Mirzaei, H. R. (2011). Effects of education based on the 
health belief model on screening behavior in high risk women for breast cancer, Tehran, Iran. Asian Pac $J$ Cancer Prev, 12(1), 49-54.

Hazavehei, S. M., Taghdisi, M. H., \& Saidi, M. (2007). Application of the Health Belief Model for osteoporosis prevention among middle school girl students, Garmsar, Iran. Educ Health (Abingdon), 20(1), 23.

Heshmati, H., Behnampour, N., Binaei, G., \& Khajavai, S. (2014). Determinants of Behavior of Students as Pedestrian and Car Occupants in Relation to Traffic Laws in 2013, Gorgan, Iran; An Application of Health Belief Model. Bull Emerg Trauma, 2(3 JUL), 115-120.

Jaber-Gaderi, N., Babaie, A., Nori, K., Zadmir, N., Nori, R., Kazemi, M., et al. (2008). [Frequency of life traumatic events and their psychological im pacts in 7-15 years old urban students of Kermanshah city]. $J$ Kerman Univ Med Sci, 12(2), 190-201.

Jackson, C. (1997). Behavioral science theory and principles for practice in health education. Health Educ Res, 12(2), 143-150.

Joshi, M. S., Beckett, K., \& Macfarlane, A. (1994). Cycle helmet wearing in teenagers-do health beliefs influence behaviour? Arch Dis Child, 71(6), 536-539.

Kealey, K. A., Ludman, E. J., Marek, P. M., Mann, S. L., Bricker, J. B., \& Peterson, A. V. (2009). Design and implementation of an effective Telephon conseling intervention for adolescent smoking cessation. $J$ Natle Cancer last, 101.

Moodi, M., Mood, M. B., Sharifirad, G. R., Shahnazi, H., \& Sharifzadeh, G. (2011). Evaluation of breast self-examination program using Health Belief Model in female students. J Res Med Sci, 16(3), 316-322.

Morowatisharifabad, M. A., Momeni Sarvestani, M., Barkhordari Firoozabadi, A., \& Fallahzadeh, H. (2012). Predictors of unsafe driving in Yazd City, Based on protection motivation theory in 2010. Horiz Med Sci, 17(3), 49-59.

Naghavi, M., \& Akbari, M. (2002). [Epidemiology injuries caused by external causes (accidents)]. Tehran: Barg Rizwan Publications.

Nazari, M., Heidarnia, A., Eftekhar Ardebili, H., Mobasheri, M., Amin Shokravi, F., \& Niknami, S. H. (2008). [Interventions based on Precede-Proceed for promoting safety behaviors in primary school boys]. Armaghan-e-danesh, 13(2), 63-93.

Nordberg, E. (2000). Injuries as a public health problem in sub-Saharan Africa: Epidemiology and prospects for control. East Afr Med J, 77(12 Suppl), S1-43.

Puvanachandra, P., Hoe, C., El-Sayed, H. F., Saad, R., Al-Gasseer, N., Bakr, M., \& Hyder, A. A. (2012). Road traffic injuries and data systems in Egypt: addressing the challenges. Traffic Inj Prev, 13 Suppl 1, 44-56. http://dx.doi.org/10.1080/15389588.2011.639417

Rezapur Shahkolai, F., Farhadi, Z., Bashirian, S., \& Roshanaei, G. H. (2013). Factors associated with school injury prevention among students in junior high schools of Famenin County, based on Health Belief Model. Research Project. Res Deput Hamadan Univ Med Sci.

Rosenstock, I. M., \& Stretcher, V. (2008). The Health Belief Model. In K. Glanz, F. M. Lewis \& B. K. Rimer (Eds.), Health Behavior and Health Education: Theory, Research, and Practice (4th ed.). San Francisco: Jossey- Bass publisher.

Sadeghnejad, F. (2014).J The impact of Precede - Proceed Model developed using Parallel Process Model (EPPM) in safety belt use among drivers Tehran]. Tehran.

Shahnazi, H., Sabooteh, S., Sharifirad, G., Mirkarimi, K., \& Hassanzadeh, A. (2015). The impact of education intervention on the Health Belief Model constructs regarding anxiety of nulliparous pregnant women. $J$ Educ Health Promot, 4, 27. http://dx.doi.org/10.4103/2277-9531.154120

Sharma, B. R. (2008). Road traffic injuries: A major global public health crisis. Pub Health, 122(12), 1399-1406. http://dx.doi.org/10.1016/j.puhe.2008.06.009

Shojaezadeh, D., Naeimi, M., Noori, K., \& Khalili Haghverdi, A. (2015). Survey of the Effect of Education Based on the Health Belief Model (HBM) on Knowledge, Perception and Performance about Having Breakfast of Primary School Student boys. J Health Hygiene, 6(2), 141-154.

Sim, T. P., \& Ng, K. C. (2005). Childhood injuries: Prevention is always better than cure. Singapore Med J, 46(3), 103-105. 
Tavassoli, E., Reisi, M., Javadzade, S. H., Gharlipour, Z., Gilasi, H. R., \& Ashrafi Hafez, A. (2015). The effect of education on improvement of intake of fruits and vegetables aiming at preventing cardiovascular diseases. Med J Islam Repub Iran, 29, 183.

Vojdani-Aram, F., Roshanaei, G. H., Hazavehei, S. M. M., Karimi-Shahanjarini, A., \& Rezapur-Shahkolai, F. (2015). [Evaluating a Health Belief Model-Based Educational Program for School Injury Prevention among Hard-of-Hearing/Deaf High School Students]. J Educ Commun Health, 1(4), 32-42.

Workagegn, F., Kiros, G., \& Abebe, L. (2015). Predictors of HIV-test utilization in PMTCT among antenatal care attendees in government health centers: institution-based cross-sectional study using health belief model in Addis Ababa, Ethiopia, 2013. HIV AIDS (Auckl), 7, 215-222. http://dx.doi.org/10.2147/hiv.s82000

Zargar, M., Sayyer Roudsarib, B., Shadman, M., \& Kavianie and Payam Tarighib, A. (2003). Pediatric transport related injuries in Tehran: The necessity of implementation of injury prevention protocols. Inj, 34(11), 820-824.

\section{Copyrights}

Copyright for this article is retained by the author(s), with first publication rights granted to the journal.

This is an open-access article distributed under the terms and conditions of the Creative Commons Attribution license (http://creativecommons.org/licenses/by/3.0/). 